\title{
VORTEX DYNAMICS IN BOSE-EINSTEIN CONDENSATES: NUMERICAL CALCULATIONS
}

\author{
D. M. Jezek ${ }^{1}$ and H. M. Cataldo \\ Consejo Nacional de Investigaciones Científicas y Técnicas \\ and \\ Departamento de Física, Facultad de Ciencias Exactas y Naturales, Universidad de \\ Buenos Aires \\ Ciudad Universitaria, (1428) Buenos Aires, Argentina
}

\begin{abstract}
We numerically study properties of the dynamics of vortices in nonrotating BoseEinstein condensates in the Thomas-Fermi regime. On the one hand, we compute the vortex energy as a function of its position and we predict, using the expression of the Magnus force, the vortex precession velocity. On the other hand, we calculate the temporal evolution of the vortex-state and test the accuracy of the previous prediction. We also investigate the validity of analytical formulae of this velocity involving the healing length. In addition, we analyze the velocity field and the angular momentum and we compare them to available analytical expressions.
\end{abstract}

PACS numbers: 03.75.Lm, 03.75.Hh, 03.75.Kk

\footnotetext{
${ }^{1}$ e-mail: djezek@df.uba.ar
} 
In a two-dimensional Bose-Einstein condensate confined by a nonrotating axisymmetric trap, the nondissipative dynamics of a single off-axis vortex consists of a precession movement [1]. On the other hand, in addition to the ordinary circulating velocity field of such a vortex, which would constitute the whole field if the vortex were located at the trap axis, there exists an induced velocity field [2]. Such an induced field, evaluated near the vortex position, defines the so-called vortex background velocity, which is closely related to the precession velocity. However, in an inhomogeneous medium the above precession velocity has been recently shown to comprise two contributions $[3,4]$ : one in fact corresponding to the background velocity and the second, which may be called a 'core' velocity, depending on the features of the vortex core.

In the present work we numerically analyze these issues for condensates confined by two different trapping potentials. On the one hand, we consider the commonly utilized harmonic potential and, on the other hand, we utilize a recently proposed polynomial potential that can sustain locally stable off-axis vortices, yielding a wider range of precession velocity values.

\section{TRAPPING POTENTIALS}

The harmonic potential has the usual form $V_{a}(r, z)=\frac{1}{2} m \omega_{r}^{2} r^{2}+\frac{1}{2} m \omega_{z}^{2} z^{2}$, where $\omega_{r}$ and $\omega_{z}$ denote the radial and axial frequencies, respectively, and $m$ is the particle mass. And the polynomial potential reads [5]:

$$
V_{b}(r, z)=\frac{1}{2} m \omega_{r}^{2} r^{2} \frac{\left(r-r_{1}\right)\left(r-r_{2}\right)}{r_{1} r_{2}}+\frac{1}{2} m \omega_{z}^{2} z^{2}
$$

where $r_{1}$ and $r_{2}$ denote numerical parameters allowing to model the shape of the potential. We have worked in the limit $\omega_{z}>>\omega_{r}$ and thus the wavefunction can be 
factorized in the $r$ and $z$ cylindrical coordinates as the product $\Psi(\mathbf{r})=\Psi(r) f(z)$, being $f(z)$ a Gaussian function [6]. In particular, we have fixed $\omega_{r}=2 \pi \times 100 \mathrm{~Hz}$ and $\omega_{z}=200 \omega_{r}$. Our condensates are formed by Rubidium atoms with a number of particles $N=10^{5}$. The function $\Psi(r)$ is obtained by solving the 2D Gross-Pitaevskii (GP) equation:

$$
\left(-\frac{\hbar^{2} \nabla^{2}}{2 m}+V_{\text {trap }}(r, 0)+g_{2 D}|\Psi(r)|^{2}\right) \Psi=\mu \Psi(r),
$$

where the effective two-dimensional coupling constant between the atoms is $g_{2 D}=$ 0.34 for the present system. In Fig. 1 (a) and (b) we show the reduced ground state densities $\rho(r)=|\Psi(r)|^{2}$ for both trapping potentials. The number of particles is large enough to assume that the Thomas Fermi (TF) approximation is appropriate to describe the ground state density, which thus may be obtained by neglecting the kinetic term in the above expression. Therefore, the shape of this function has the form of the respective inverted potentials. On the other hand, the vortex states are numerically obtained by phase imprinting methods. The corresponding density profiles $\rho_{v}(r)$ (Fig. 1) are similar to that of the ground state $\rho_{0}(r)$, except for the presence of the vortex core.

\section{VORTEX PRECESSION VELOCITY IN INHOMOGENEOUS} MEDIA

When the energy $E$ depends on the vortex position $\mathbf{r}_{0}$, the vortex velocity $\mathbf{v}_{p}$ may be extracted from the following expression [7], related to the Magnus Force [8]:

$$
2 \pi \hbar \rho_{0}\left(r_{0}\right)\left(\hat{\mathbf{z}} \times \mathbf{v}_{p}\right)=\nabla E\left(r_{0}\right)
$$

being $r_{0}=\left|\mathbf{r}_{0}\right|$. For this purpose we have numerically computed the energy $E$ as a 
function of the vortex position for both trapping potentials. In Fig. 2 we depict the vortex energy $E_{v}=E-E_{0}$, being $E_{0}$ the ground state energy. We also plot in that figure a theoretical estimate of the vortex energy $E_{v}^{\text {theor }}$, which is displayed as solid lines. Such an estimate has been derived $[9,10]$ under the hypothesis that the size of the vortex core is of the order of the healing length $\xi$, which is utilized as a cutoff parameter in the corresponding integral. Thus, the theoretical prediction reads

$$
E_{v}^{\text {theor }}\left(r_{0}\right)=\frac{\pi \hbar^{2}}{m} \rho_{0}\left(r_{0}\right) \ln \left(R_{T F} / \xi\right)
$$

where $R_{T F}$ denotes the condensate radius and $\xi=\sqrt{\hbar^{2} /\left(2^{3 / 2} m g_{2 D} \rho_{0}\left(r_{0}\right)\right)}$. Figure 3 shows the vortex precession velocity as a function of the vortex position, calculated from Eq. (3), for both trapping potentials. We have also performed numerical simulations of the time evolution of the system, in order to obtain the exact precession velocity, for several initial conditions marked as star dots in the same graph. It may be observed that the prediction extracted from the numerically obtained energy values shows an excellent agreement with the exact precession velocity.

\section{VORTEX VELOCITY FIELD}

The velocity field induced by a single vortex in an inhomogeneous condensate is called the background velocity $\mathbf{v}_{B}(\mathbf{r})$. This velocity does not include the divergent part due to the vortex itself, that is, if $\mathbf{v}(\mathbf{r})$ denotes the total velocity field, then

$$
\mathbf{v}_{B}(\mathbf{r})=\mathbf{v}(\mathbf{r})-\frac{\hbar}{m} \frac{1}{\left|\mathbf{r}-\mathbf{r}_{0}\right|^{2}} \hat{\mathbf{z}} \times\left(\mathbf{r}-\mathbf{r}_{0}\right) .
$$

Sheehy and Radzihovsky [2] have derived the following approximate expression valid near the vortex core

$$
\mathbf{v}_{B}(\mathbf{r})^{\text {theor }} \simeq \frac{\hbar}{2 m} \frac{\hat{\mathbf{z}} \times \nabla \rho_{0}\left(r_{0}\right)}{\rho_{0}\left(r_{0}\right)} \ln \left(\frac{\left|\mathbf{r}-\mathbf{r}_{0}\right|\left|\nabla \rho_{0}\left(r_{0}\right)\right|}{2 \rho_{0}\left(r_{0}\right)}\right),
$$


which was obtained under the assumption $\nabla \cdot \mathbf{j} \equiv 0$, being $\mathbf{j}$ the particle current density. Now, taking into account the continuity equation, we realize that such an assumption could only be valid outside the vortex core, where $\partial \rho_{v} / \partial t=0$. Thus, Eq. (6) should be utilized up to the border of the core at most, but not inside it, where $\partial \rho_{v} / \partial t \neq 0$. In Fig. 4 we depict the theoretical estimate (6) together with our numerical results. We may see that the theoretical prediction yields lower values than the numerical results for the trapping potential $V_{a}$, whereas it yields for $V_{b}$ a rather large overestimate.

\section{RELATION BETWEEN THE BACKGROUND VELOCITY AND THE VORTEX PRECESSION VELOCITY}

It is well known that a vortex moves with the background velocity in a uniform superfluid. Various authors have assumed, generalizing this concept, that a vortex in an inhomogeneous system should move with the background velocity evaluated at $\left|\mathbf{r}-\mathbf{r}_{0}\right| \sim l_{c}\left(r_{0}\right)$, being $l_{c}$ the size of the core [2]. Now, if we locate the border of the core where the form factor acquires the value $F \simeq 0.95$ (see Fig. 4), we obtain $l_{c} \simeq 5 \xi\left(r_{0}\right)$ from our numerical results. Thus we have also calculated according to this procedure such a background velocity for all the vortex positions denoted as star dots in Fig. 3. We have found in all cases severe discrepancies between the background and the exact precession velocities. Particularly, for the vortex location at $x=8$ (trapping potential $V_{a}$, Figs. 3 and $4(\mathrm{a})$ ) we have obtained a precession velocity $\simeq 0.34$ and a background velocity $\simeq 0.23$, while for a vortex at $x=-8.5$ (trapping potential $V_{b}$, Figs. 3 and $4(\mathrm{~b})$ ) the precession velocity is $\simeq 0.11$ and the background velocity is $\simeq 0.05$. Such discrepancies were recently predicted by Nilsen et al. [3], who proposed that in order to obtain a better approximation to the 
precession velocity than that of the background, a corrective term should be added to the latter as follows,

$$
\mathbf{v}_{p} \simeq \mathbf{v}_{B}-f \frac{\hbar}{2 m} \frac{\hat{\mathbf{z}} \times \nabla \rho_{0}}{\rho_{0}}
$$

where $f=1$ and the last term corresponds to a 'core' contribution [3] to the precession velocity. The proposal of Ref. [3] was later numerically tested for the harmonic trapping potential [4] finding that $f=2$ was actually the best figure to reproduce the results. Now the present study confirms that the expression (7) with $f=2$ yields the best agreement with the numerical results for both trapping potentials.

\section{ANGULAR MOMENTUM}

Finally, we have also numerically investigated the accuracy of an available formula for the angular momentum of an off-axis vortex [11]:

$$
L_{z}\left(r_{0}\right)=\hbar \int_{r_{0}}^{\infty} 2 \pi r d r \rho_{0}(r)
$$

finding an excellent agreement with the numerical results for both trapping potentials, as seen in Fig. 5.

\section{CONCLUDING REMARKS}

We shall summarize in the following the main conclusions of the present study. Firstly, we have seen that the vortex velocity value arising from the balance between the Magnus force and the force stemming from the gradient of the vortex energy, constitutes a very good estimate of the vortex precession velocity. On the other hand, we have found that previously reported formulae for the background velocity, yield values that may substantially differ from numerically computed ones. In addition, 
we have shown that such a velocity differs appreciably from the vortex precession velocity through a 'core' contribution, in accordance with our previous findings. Finally, we have encountered that a previously reported analytical estimate of the angular momentum turns out to be a very accurate one.

\section{ACKNOWLEDGMENTS}

This work has been performed under Grant PIP 5409 from CONICET, Argentina.

\section{References}

[1] A. L. Fetter and A. A. Svidzinsky, J. Phys.: Condens. Matter 13, R135 (2001).

[2] D. E. Sheehy and L. Radzihovsky, Phys. Rev. A 70, 063620 (2004).

[3] H. M. Nilsen, G. Baym, and C. J. Pethick, Proc. Natl. Acad. Sci. U.S.A. 103, 7978 (2006).

[4] D. M. Jezek and H. M. Cataldo, Phys. Rev. A77, 043602 (2008).

[5] D. M. Jezek, P. Capuzzi, and H. M. Cataldo, J. Phys. B: At. Mol. Opt. Phys. 41, 045304 (2008).

[6] Y. Castin and R. Dum, Eur. Phys. J. D 7, 399 (1999).

[7] S. A. McGee and M. J. Holland, Phys. Rev. A 63, 042608 (2001).

[8] R. J. Donnelly, Quantized Vortices in Helium II (Cambridge University Press, Cambridge, 1991). 
[9] E. Lundh and P. Ao, Phys. Rev. A 61, 063612 (2000).

[10] A. L. Fetter, Phys. Rev. A 69, 043617 (2004).

[11] M. Guilleumas and R. Graham, Phys. Rev. A64, 033607 (2001). 


\section{FIGURE CAPTIONS}

FIGURE 1: Section of the particle density at $y=0$ for the trapping potentials $V_{a}$ in (a) and $V_{b}$ in (b). The abscisa is given in units of the harmonic oscillator length $l_{r}=\sqrt{\hbar /\left(m \omega_{r}\right)}$. The solid lines correspond to the respective ground states, while the dotted lines correspond to states with a vortex located at $x / l_{r}=8$ and $y / l_{r}=0$ in (a), and $x / l_{r}=-8.5$ and $y / l_{r}=0$ in (b). We have set $r_{1}=10 l_{r}$ and $r_{2}=18 l_{r}$ in the expression of $V_{b}$ (Eq. (1)).

FIGURE 2: Vortex energy in units of $\hbar \omega_{r}$ as a function of the vortex position $\mathbf{r}_{\mathbf{0}}=(x, 0)$ for the trapping potentials $V_{a}$ in (a) and $V_{b}$ in (b). The dots correspond to the numerical results, while the solid lines correspond to the theoretical estimate $(4)$.

FIGURE 3: Vortex precession velocity in units of $l_{r} \omega_{r}$ as a function of the vortex position along the $x$-axis. Solid lines and dots correspond to values extracted from Eq. (3) with respective energy values arising from Fig. 2. The star dots correspond to values arising from numerical simulations of the time evolution of each initial configuration.

FIGURE 4: Background velocity field in units of $l_{r} \omega_{r}$ at $y=0$. The filled dots correspond to numerical results, while the solid lines correspond to the theoretical estimate (6). The dots-plus-dotted lines correspond to the form factor $F=\rho_{v} / \rho_{0}$ (arbitrary scales), while the vertical dashed lines show the limits of the core region. (a): Vortex located at $x / l_{r}=8$ and $y / l_{r}=0$ with the trapping potential $V_{a}$. (b): Vortex located at $x / l_{r}=-8.5$ and $y / l_{r}=0$ with the trapping potential $V_{b}$.

FIGURE 5: Angular momentum in units of $\hbar$ as a function of the vortex position along the $x$-axis for the trapping potentials $V_{a}$ in (a) and $V_{b}$ in (b). The dots correspond to the numerical results, while the solid line corresponds to Eq. (8). 


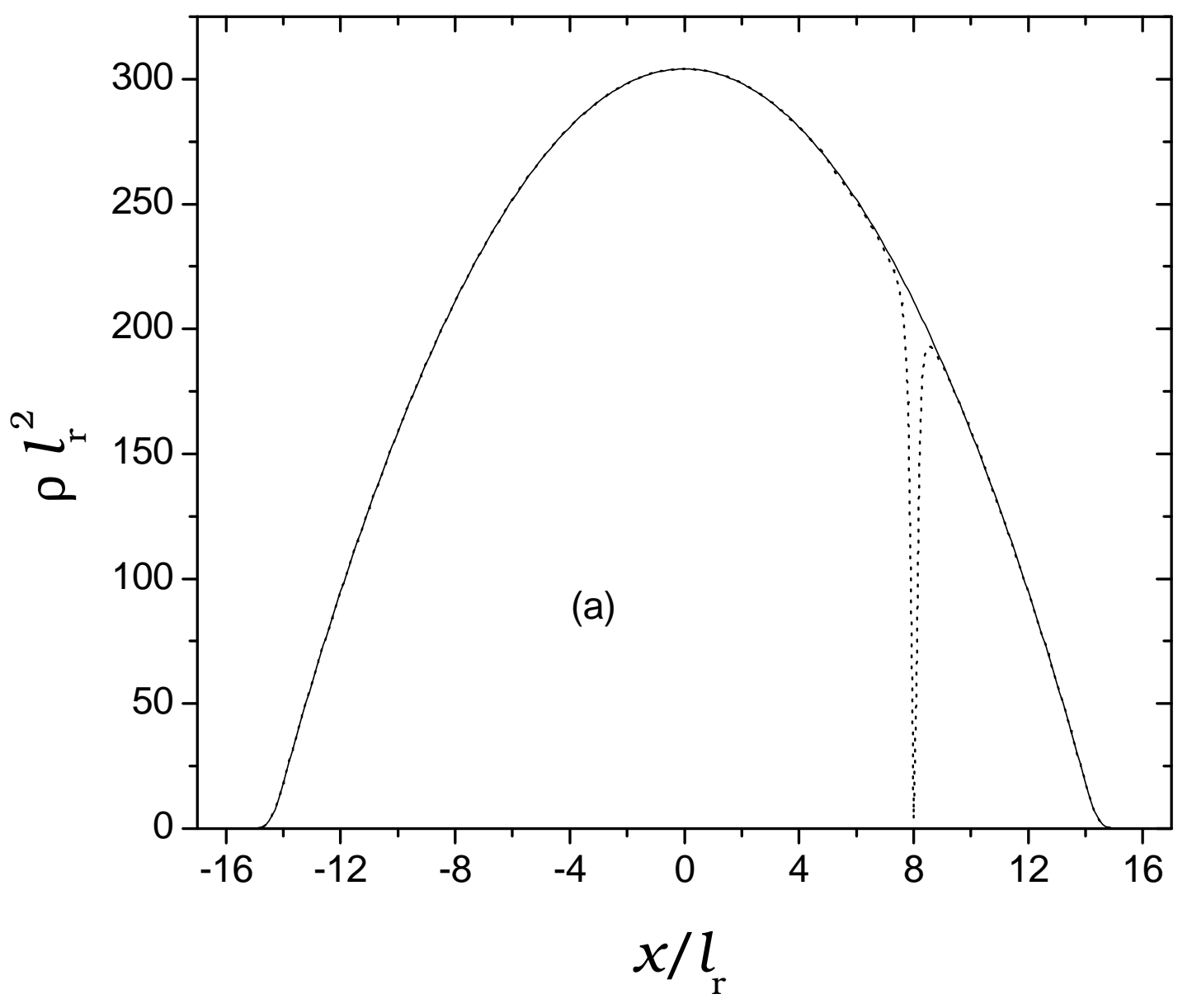

Jezek, VORTEX DYNAMICS IN BOSE-EINSTEIN CONDENSATES, Figure 1 (a) 


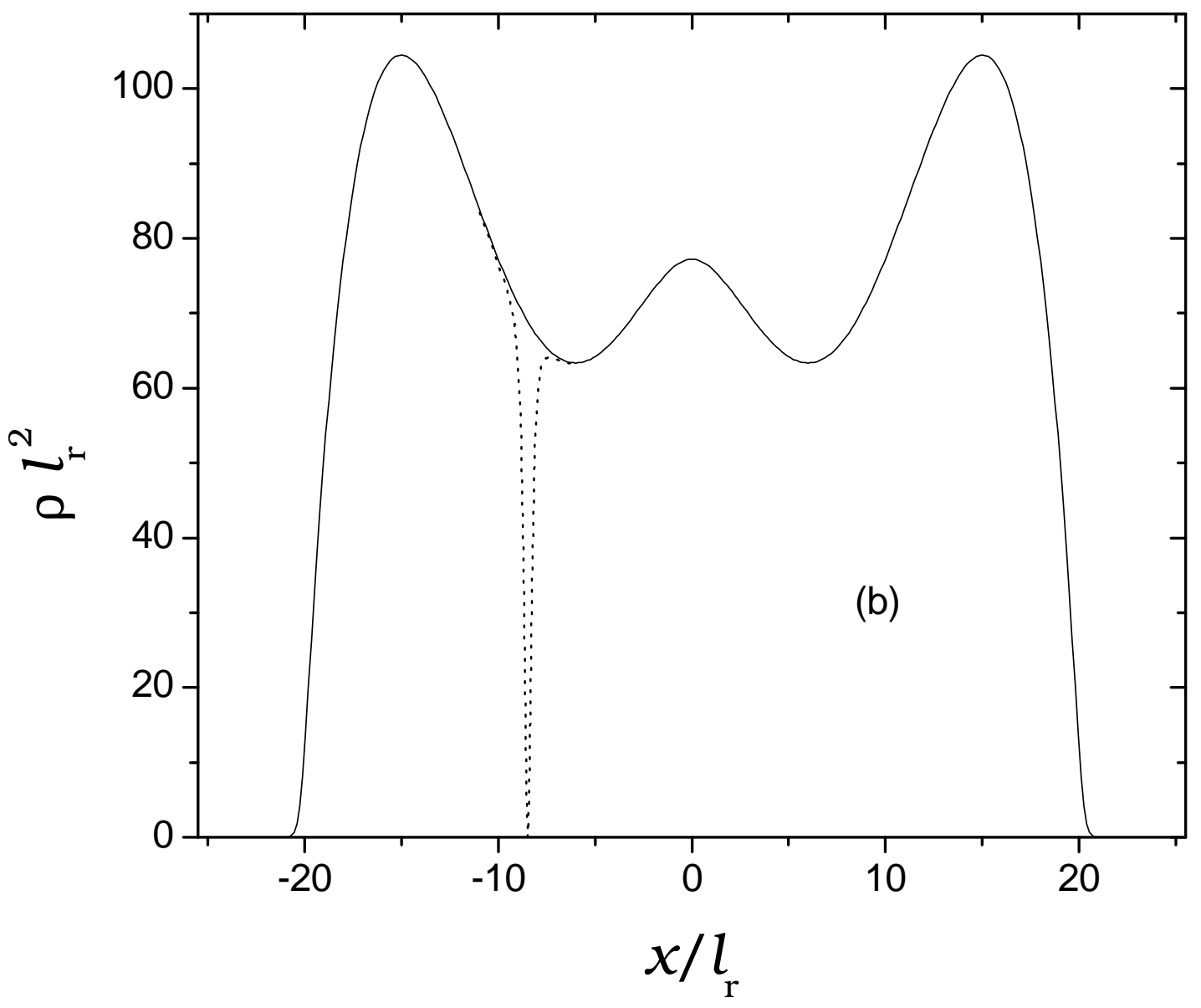

Jezek, VORTEX DYNAMICS IN BOSE-EINSTEIN CONDENSATES, Figure 1 (b) 


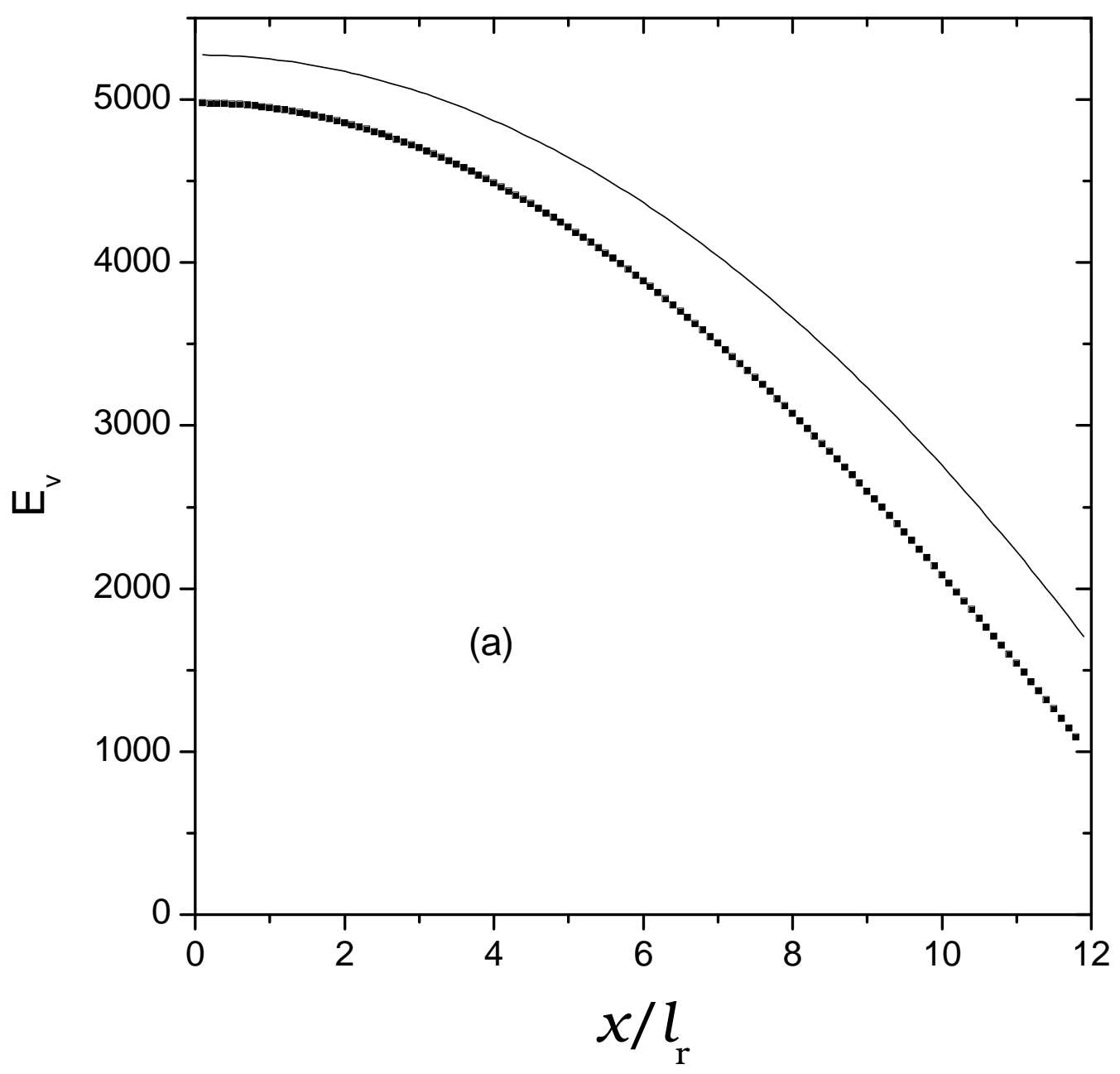

Jezek, VORTEX DYNAMICS IN

BOSE-EINSTEIN CONDENSATES, Figure 2 (a) 


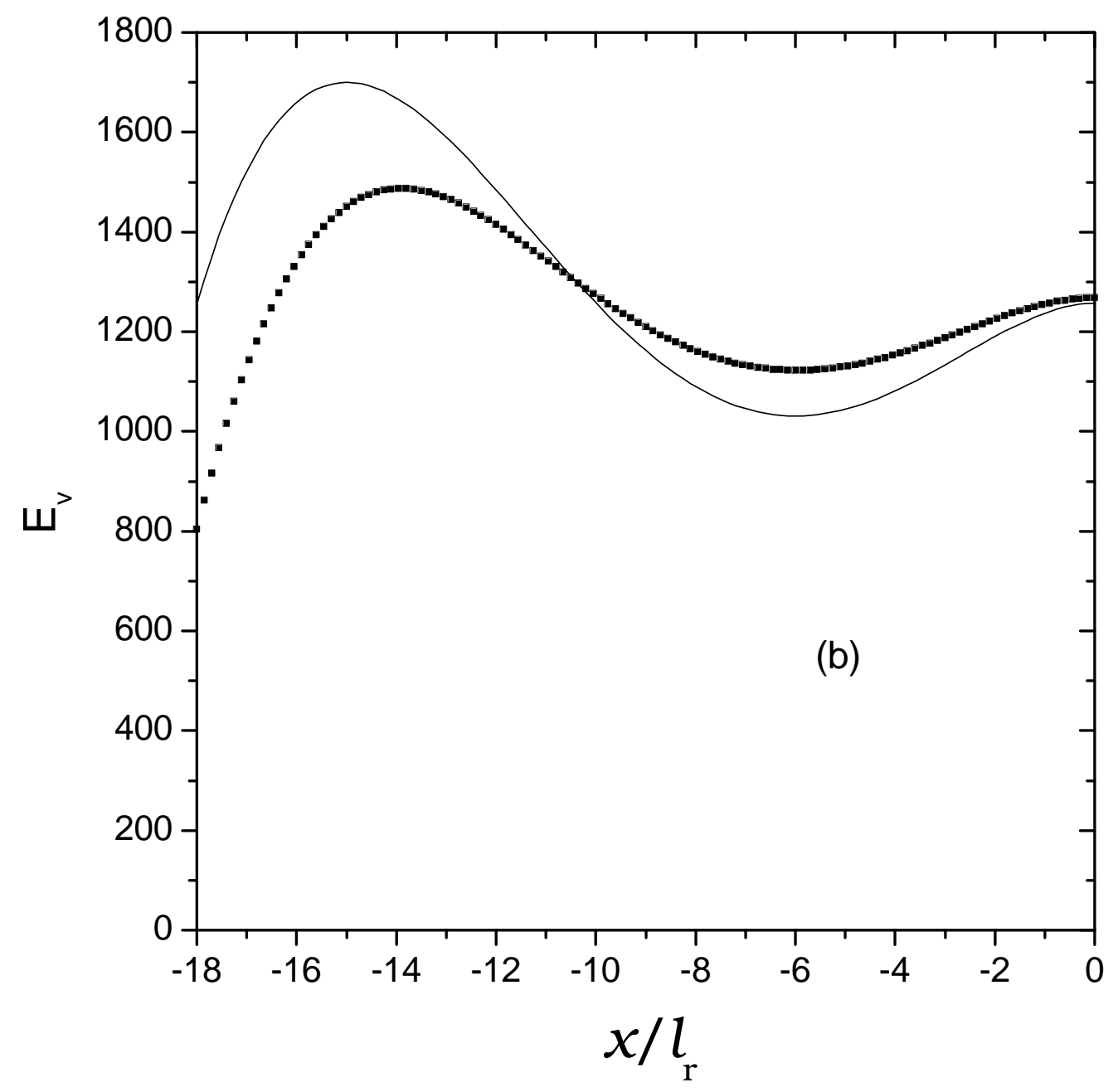

Jezek, VORTEX DYNAMICS IN BOSE-EINSTEIN CONDENSATES, Figure 2 (b) 


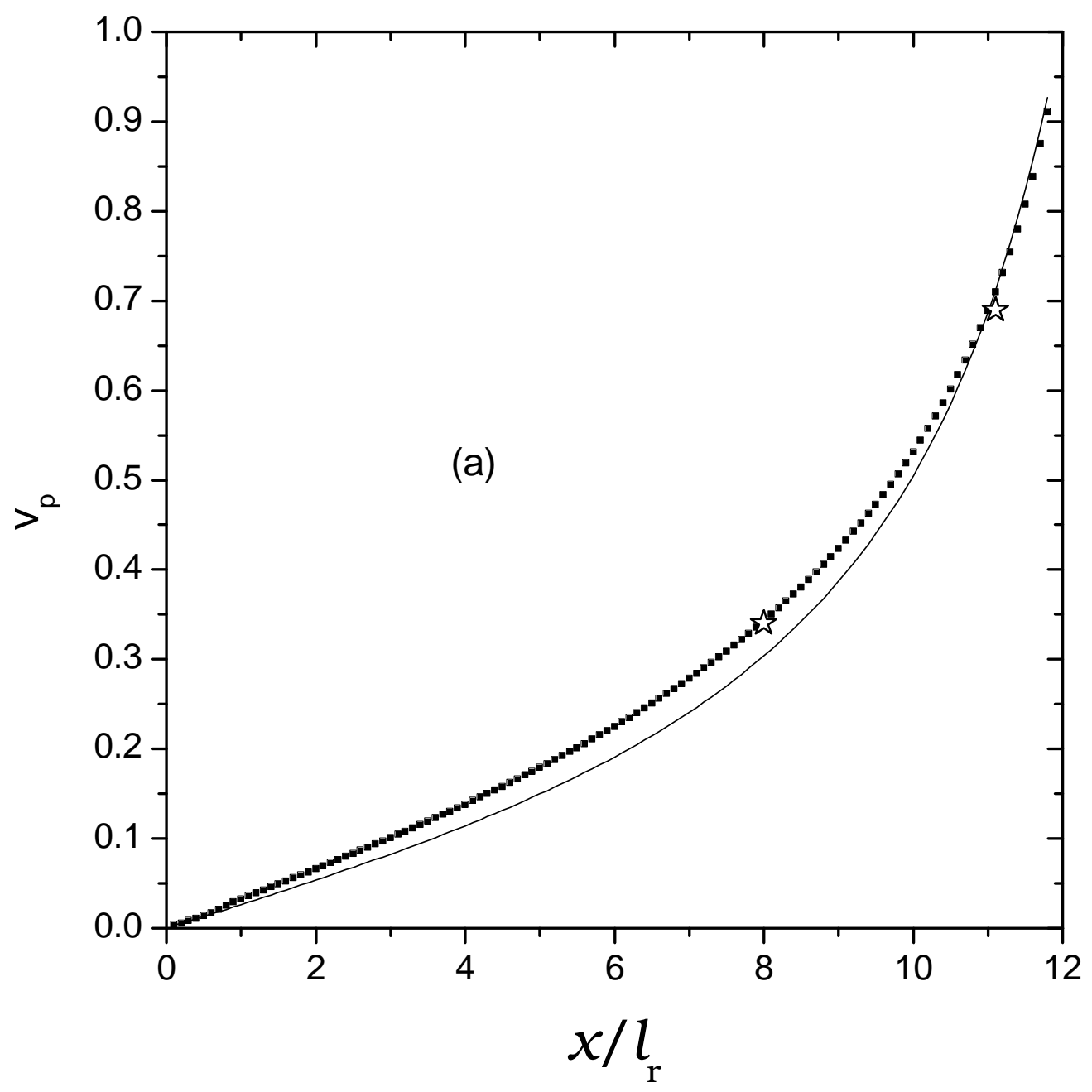

Jezek, VORTEX DYNAMICS IN BOSE-EINSTEIN CONDENSATES, Figure 3 (a) 


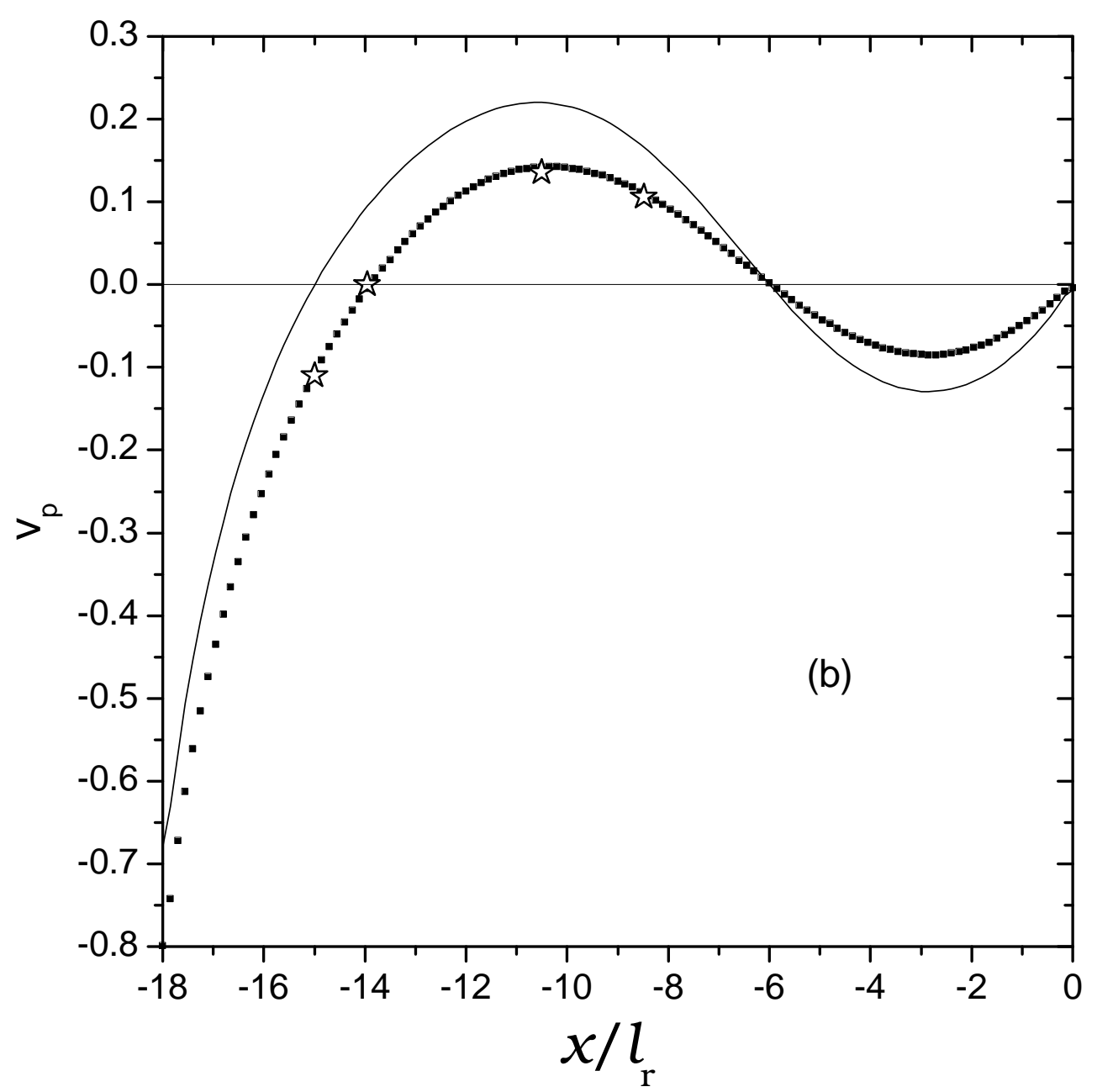

Jezek, VORTEX DYNAMICS IN BOSE-EINSTEIN CONDENSATES, Figure 3 (b) 


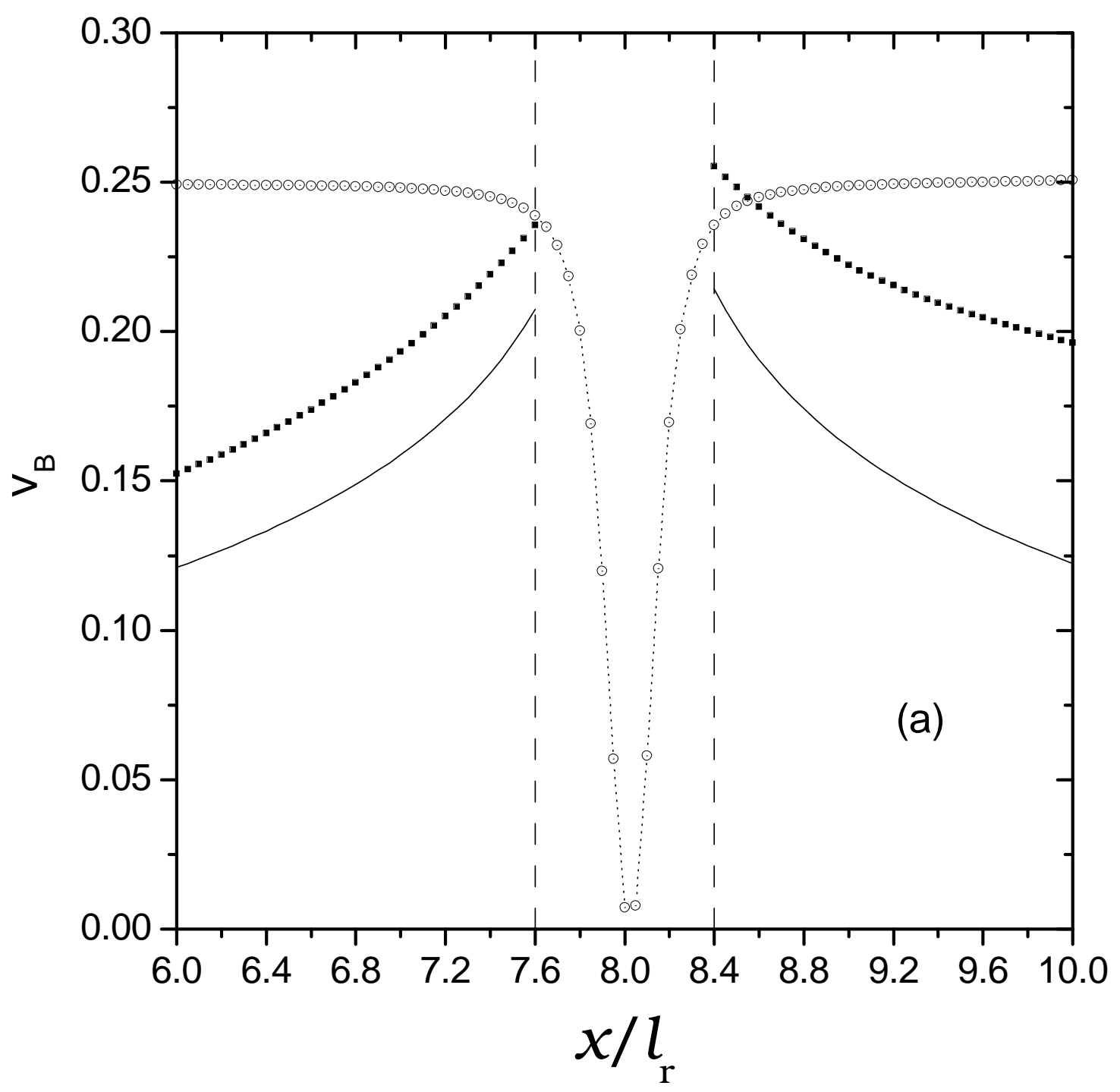

Jezek, VORTEX DYNAMICS IN BOSE-EINSTEIN CONDENSATES, Figure 4 (a) 


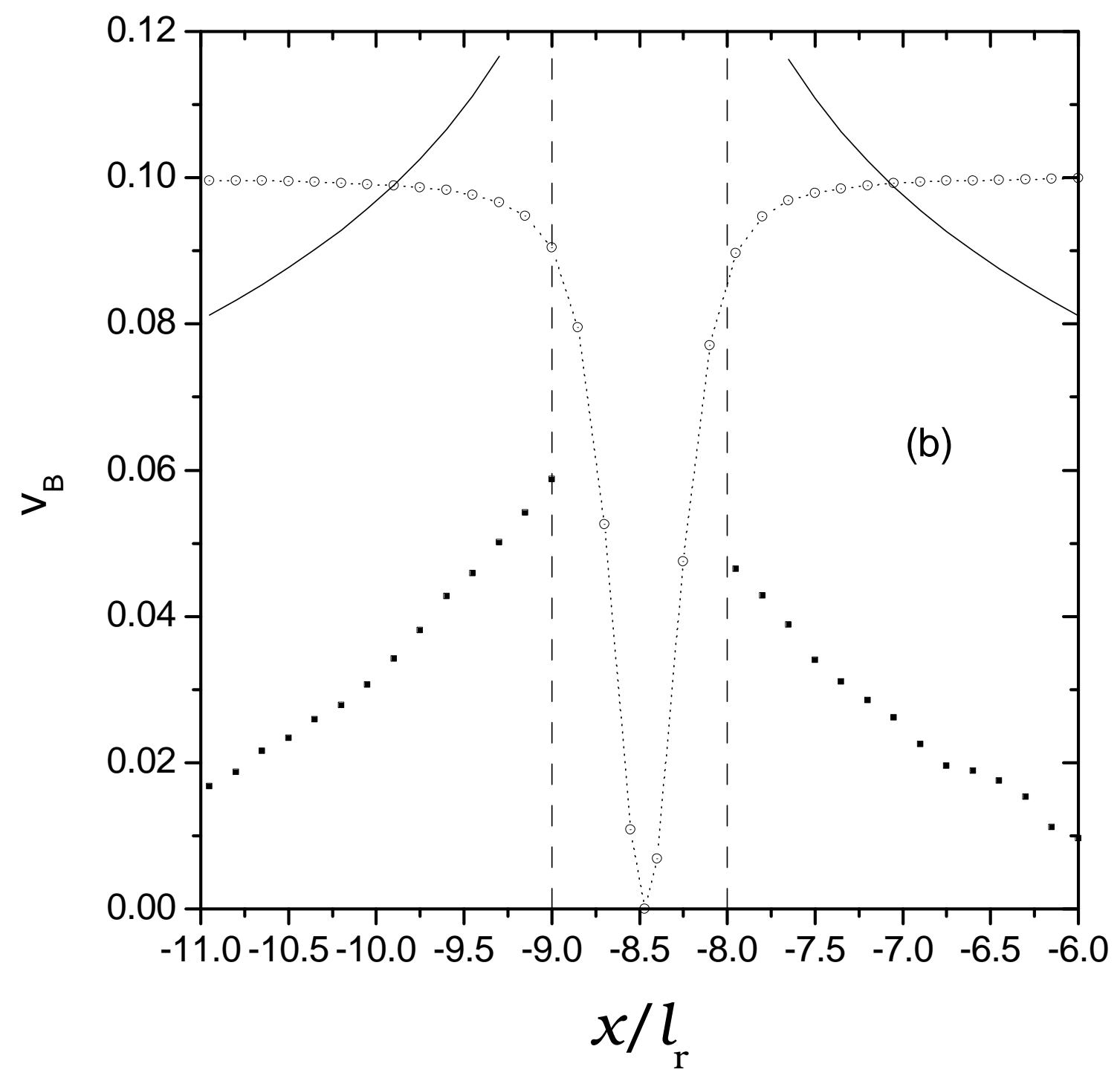

Jezek, VORTEX DYNAMICS IN BOSE-EINSTEIN CONDENSATES, Figure 4 (b) 


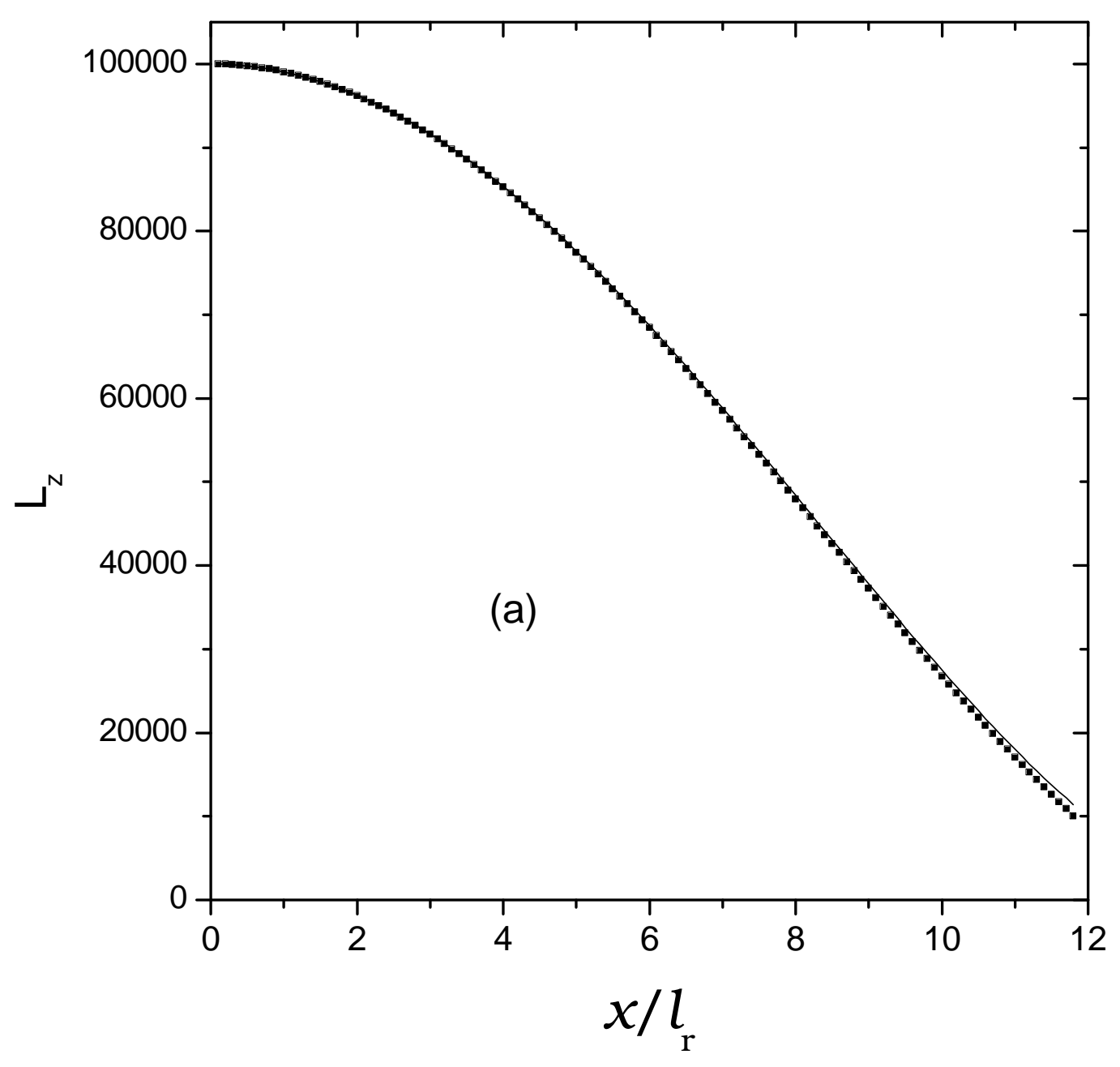

Jezek, VORTEX DYNAMICS IN BOSE-EINSTEIN CONDENSATES, Figure 5 (a) 


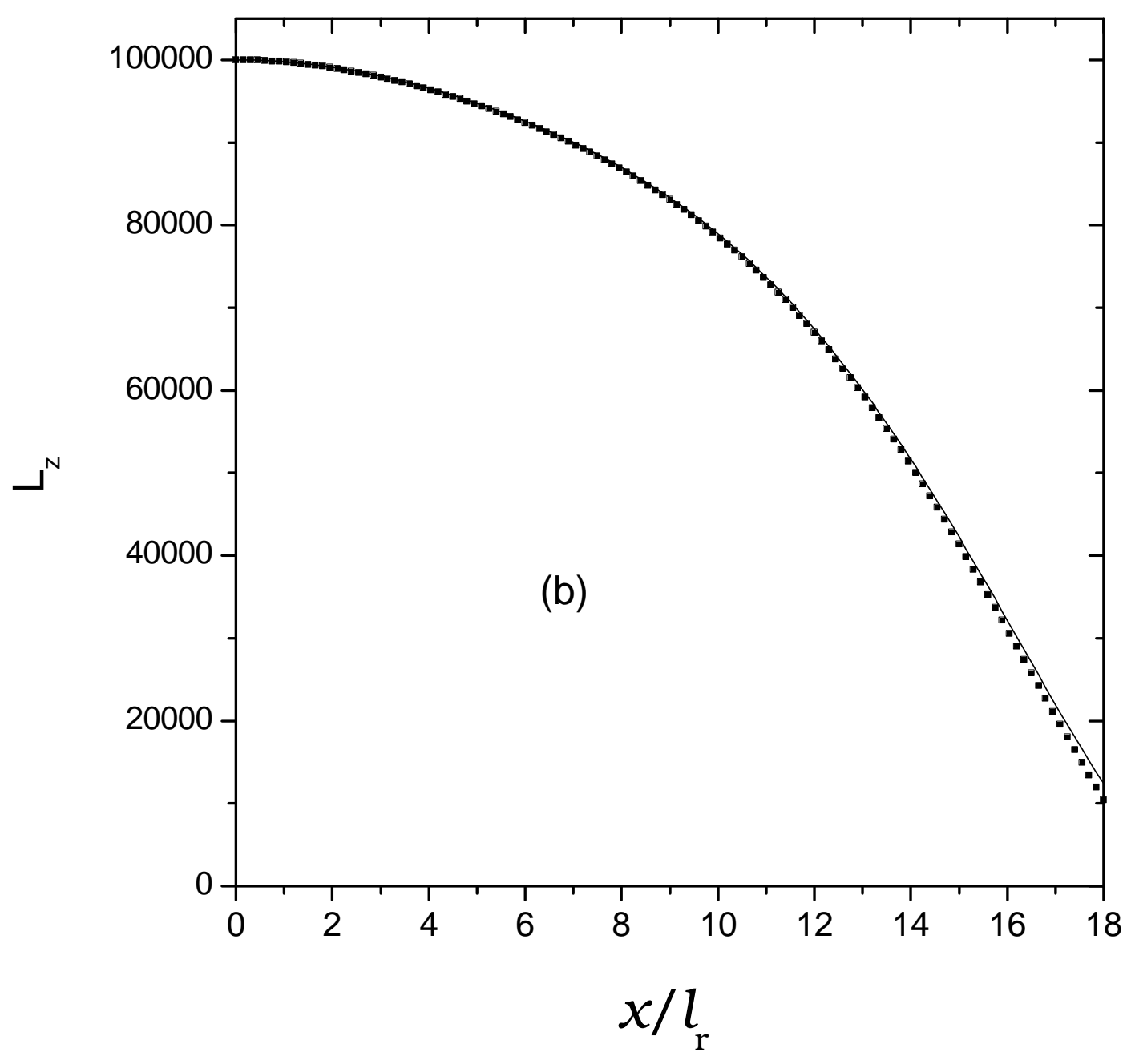

Jezek, VORTEX DYNAMICS IN BOSE-EINSTEIN CONDENSATES, Figure 5 (b) 